\title{
Synthesis, Analgesic and Antimicrobial Activities of Some Novel Isoxazole Derivatives
}

\author{
S.K. Sahu' ${ }^{1}$, M. Banerjee ${ }^{1}$, D. Sahu1 ${ }^{1}$, C.C. Behera ${ }^{1}$, G.C. Pradhan² \\ and Md. Afzal Azam ${ }^{3}$ \\ ${ }^{1}$ University Department of Pharmaceutical Sciences, Utkal University, Bhubaneswar-751004, India. \\ ${ }^{2}$ Department of Chemistry, Utkal University, Bhubaneswar-751004, India. \\ ${ }^{3}$ Department of Pharmaceutical Chemistry, J. S. S., College of Pharmacy, Tamil Nadu, \\ Ootacamund-643 001, India.
}

\begin{abstract}
Substituted aryl-N-chalconyl aminophenols 1a-f were synthesized by base catalysed condensation of equimolar mixture of N-(4-hydroxyphenyl)-acetamide and appropriate araldehydes. Treatment of compounds 1a-f with hydroxylamine hydrochloride in ethanol afforded a series of novel 4-(5'-substituted-aryl-4',5'-dihydroisoxazole-3'yl-amino) phenols have been synthesized by treating substituted aryl-N-chalconyl aminophenol with hydroxylamine hydrochloride. Structures of newly synthesized compounds $2 \mathrm{a}-\mathbf{f}$ were confirmed by IR, ${ }^{1} \mathrm{H}-\mathrm{NMR}$ and elemental analysis data. The synthesized compounds were investigated for their analgesic and antimicrobial activities. Compounds $2 \mathbf{e}$ and $\mathbf{2 f}$ exhibited significant analgesic activity in comparison to the reference drug paracetamol. In in vitro anti-microbial screening, compounds $\mathbf{2 c}$ and $\mathbf{2 f}$ showed higher antibacterial and antifungal activity in comparison to the reference standard ciprofloxacin and clotrimazole, respectively. Compound $\mathbf{2 f}$ bearing 4-Cl phenyl substitution at 5 position of Ioxazoline ring was found to be the most potent compound of the series.
\end{abstract}

Key words: Isoxazole, Analgesic, Antibacterial activity.

\section{INTRODUCTION}

The prevalence of isoxazole cores in natural and biologically active molecules has stimulated the need for elegant and efficient ways to make these heterocyclic lead. The treatment of pain continues to be the subject of considerable pharmaceutical and clinical research, recent years; isoxazoles are of great interest due to their exceptional biological activities. A systematic investigation of this class of heterocycle revealed that isoxazole containing pharmacoactive agents play important role in medicinal chemistry. Microbial infections often produce pain and inflammation. Chemotherapeutic, analgesic and anti-

Correspondence to: S. K. Sahu

E-mail: tutu_kh@yahoo.com

Dhaka Univ. J. Pharm. Sci. 7(2): 113-118, 2008 (December) inflammatory drugs are prescribed simultaneously in normal practice. The compound possessing all activities is not common. It has been reported that isoxazolines possess analgesic, anti-inflammatory ${ }^{1-4}$ and antimicrobial ${ }^{5-11}$ activities. In view of these above fact, an attempt has been made for the synthesis of novel 4-(5'-substituted-aryl-4,5'dihydro-isoxazole-3'-yl-amino) phenols possessing potent biological activities. The synthesized compounds were tested for their possible analgesic and anti-microbial activities.

\section{MATERIALS AND METHODS}

Melting points were determined in open capillaries and were uncorrected. Purity of the 
compounds was checked by TLC. IR spectra $(\mathrm{KBr})$ were recorded on a JASCO FT/ IR 410 spectrophotometer $\left(v_{\max }\right.$ in $\left.\mathrm{cm}^{-1}\right) .{ }^{1} \mathrm{H}$ NMR $\left(\mathrm{CDCl}_{3}\right)$ on a Bruker DPX $300-\mathrm{MHz}$ spectrometer using TMS as internal reference (chemical shifts in $\delta$ ppm). $\mathrm{C}, \mathrm{H}$ and $\mathrm{N}$ analyses were carried out on a Euro EA (Italy) analyzer. N-(4'hydroxy phenyl)-acetamide (99\%, Cipla Ltd.), Benzaldehyde (99 \%, Merck), Furfuraldehyde (99\%, Burgoyne Burbidges and co.), Salicylaldehyde (99\%, CDH Lab), anisaldehyde (99 $\%$, SD-fine chemicals), p-cholorobenzaldehyde (99 $\%$,Hi-media), p-nitrobenzaldehyde(99 \%, Hi-media), $\mathrm{NaOH}$ (99\%, SD-fine. chemicals), Ethanol (97\%, LOBA chemicals), Hydroxylamine hydrochloride (98 $\%$, SD-fine chemicals), Mueller Hinton agar (99\%, Hi-media), Sabouraud dextrose agar (99 \%, Hi- media). Carboxymethylcellulose

(Burgoyne Burbidges and co.)

\section{Chemistry}

Preparation of N-(4'-hydroxyphenyl)-3-phenylacrylamide 1a: To a mixture of $\mathrm{N}-(4$ 'hydroxy phenyl)-acetamide $(0.01 \mathrm{~mol})$ and benzaldehyde $(0.01 \mathrm{~mol})$ in ethanol $(50 \mathrm{ml}), 2 \% \mathrm{NaOH}$ solution (1 $\mathrm{ml}$ ) was added and stirred for $10 \mathrm{~h}$ at room temperature and then refluxed for $6 \mathrm{~h}$ on a water bath. The excess solvent was distilled off under vacuum and poured into ice-cold water. The solid 1a thus separated was filtered, dried and recrystallized from ethanol. Compounds $\mathbf{1 b}-\mathbf{f}$ were prepared similarly by using appropriate araldehydes. Melting points, yields and molecular formula are summarized in Table 1.

Table 1. Characterization of synthesized compounds 1a-f and 2a-f:

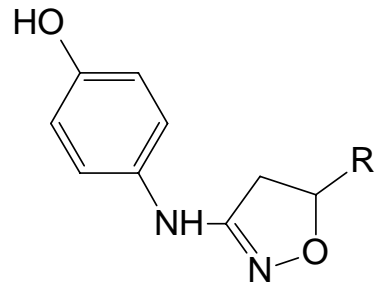

\begin{tabular}{|c|c|c|c|c|}
\hline Compound & (R) & Formula & MP $\left({ }^{0} \mathrm{C}\right)^{*}$ & Yield (\%) \\
\hline $1 \mathrm{a}$ & $-\mathrm{C}_{6} \mathrm{H}_{5}$ & $\mathrm{C}_{15} \mathrm{H}_{13} \mathrm{O}_{2} \mathrm{~N}$ & 150 & 72 \\
\hline $1 b$ & -Furyl & $\mathrm{C}_{13} \mathrm{H}_{11} \mathrm{O}_{3} \mathrm{~N}$ & 160 & 63 \\
\hline 1c & $-4-\mathrm{NO}_{2}-\mathrm{C}_{6} \mathrm{H}_{4}$ & $\mathrm{C}_{15} \mathrm{H}_{12} \mathrm{O}_{4} \mathrm{~N}_{2}$ & 108 & 81 \\
\hline 1d & $-4-\mathrm{OCH}_{3}-\mathrm{C}_{6} \mathrm{H}_{4}$ & $\mathrm{C}_{16} \mathrm{H}_{15} \mathrm{O}_{3} \mathrm{~N}$ & 152 & 74 \\
\hline $1 e$ & $-2-\mathrm{OH}-\mathrm{C}_{6} \mathrm{H}_{4}$ & $\mathrm{C}_{15} \mathrm{H}_{13} \mathrm{O}_{3} \mathrm{~N}$ & 142 & 72 \\
\hline 1f & $-4-\mathrm{Cl}-\mathrm{C}_{6} \mathrm{H}_{4}$ & $\mathrm{C}_{15} \mathrm{H}_{12} \mathrm{O}_{2} \mathrm{NCl}$ & 150 & 75 \\
\hline $2 a$ & $-\mathrm{C}_{6} \mathrm{H}_{5}$ & $\mathrm{C}_{15} \mathrm{H}_{14} \mathrm{~N}_{2} \mathrm{O}_{2}$ & 210 & 73 \\
\hline $2 \mathbf{b}$ & $-\mathrm{C}_{6} \mathrm{H}_{5}$ & $\mathrm{C}_{13} \mathrm{H}_{12} \mathrm{~N}_{2} \mathrm{O}_{3}$ & 176 & 66 \\
\hline $2 c$ & $-4-\mathrm{NO}_{2}-\mathrm{C}_{6} \mathrm{H}_{4}$ & $\mathrm{C}_{15} \mathrm{H}_{13} \mathrm{~N}_{3} \mathrm{O}_{4}$ & 120 & 78 \\
\hline 2d & $-4-\mathrm{OCH}_{3}-\mathrm{C}_{6} \mathrm{H}_{4}$ & $\mathrm{C}_{16} \mathrm{H}_{16} \mathrm{~N}_{2} \mathrm{O}_{3}$ & 190 & 73 \\
\hline $2 e$ & $-2-\mathrm{OH}-\mathrm{C}_{6} \mathrm{H}_{4}$ & $\mathrm{C}_{15} \mathrm{H}_{14} \mathrm{~N}_{2} \mathrm{O}_{3}$ & 164 & 71 \\
\hline $2 f$ & $-4-\mathrm{Cl}-\mathrm{C}_{6} \mathrm{H}_{4}$ & $\mathrm{C}_{15} \mathrm{H}_{13} \mathrm{~N}_{2} \mathrm{O}_{2} \mathrm{Cl}$ & 152 & 77 \\
\hline
\end{tabular}

1a $\left(\mathrm{R}=-\mathrm{C}_{6} \mathrm{H}_{5}\right)$ :-IR $\left(\mathrm{KBr}, \mathrm{cm}^{-1}\right): 3452(\mathrm{Ar}-\mathrm{OH}$ str.), 3301(NH str.), 3016 (C-H str.), $1650(\mathrm{C}=\mathrm{O})$, $1610(\mathrm{C}=\mathrm{C}$ str. $) ;{ }^{1} \mathrm{HNMR}(\delta \mathrm{ppm})\left(\mathrm{CDCl}_{3}+\mathrm{DMSO}-\right.$ $\left.\mathrm{d}_{6}\right): 5.35(1 \mathrm{H}, \mathrm{s}, \mathrm{Ar}-\mathrm{OH}), 6.11(1 \mathrm{H}, \mathrm{s}, \mathrm{N}-\mathrm{H}), 6.76$ $(2 \mathrm{H}, \mathrm{dd}, \mathrm{CH}), 7.10-8.00(\mathrm{~m}, 9 \mathrm{H}, \mathrm{Ar}-\mathrm{H})$; Analysis $\left(\mathrm{C}_{15} \mathrm{H}_{13} \mathrm{O}_{2} \mathrm{~N}\right.$ ) cal. (found) \%: C; 75.30 (75.52), $\mathrm{H} ; 5.48(4.98) \mathrm{N} ; 5.88$ (6.21); MS (m/z) : $239\left(\mathrm{M}^{+}\right)$.
$1 \mathbf{b}\left(\mathrm{R}=\right.$-Furyl):- IR ( $\left.\mathrm{KBr}, \mathrm{cm}^{-1}\right): 3300$ (Ar-OH str.), 3253 (NH str.), $2922\left(\mathrm{CH}_{2}\right.$ str.), $1665(\mathrm{C}=\mathrm{O}), 1476$ (C=C str.), 1137 (C-O-C str.); ${ }^{1} \mathrm{HNMR}$ ( $\delta$ ppm) $\left(\mathrm{CDCl}_{3}+\mathrm{DMSO}_{-} \mathrm{d}_{6}\right): 4.45(\mathrm{~m}, 3 \mathrm{H}, \mathrm{CH}$ furyl $), 5.65$ $(1 \mathrm{H}, \mathrm{s}, \mathrm{Ar}-\mathrm{OH}), 6.21(1 \mathrm{H}, \mathrm{s}, \mathrm{N}-\mathrm{H}), 6.76(2 \mathrm{H}, \mathrm{dd}$, $\mathrm{CH}), 7.13-8.00(\mathrm{~m}, 4 \mathrm{H}, \mathrm{Ar}-\mathrm{H})$; Analysis $\left(\mathrm{C}_{13} \mathrm{H}_{11} \mathrm{O}_{3} \mathrm{~N}\right)$ cal. (found) \%: C; 68.11 (68.43), H; 4.84 (4.49), N; 
6.11 (5.89); $\mathrm{MS}(\mathrm{m} / \mathrm{z}): 229.1 \mathrm{c}\left(\mathrm{R}=\mathrm{p}-\mathrm{NO}_{2}-\mathrm{C}_{6} \mathrm{H}_{4}\right):-\mathrm{IR}$ $\left(\mathrm{KBr}, \mathrm{cm}^{-1}\right)$ : 3490 (Ar-OH str.), 3291 (NH str.), 3099 (C-H str.), 2851( $\mathrm{CH}_{2}$ str.), 1560 (C- $\mathrm{NO}_{2}$ asym. str.), 1485(C $=\mathrm{C}$ str. $),{ }^{1} \mathrm{HNMR}(\delta \mathrm{ppm})\left(\mathrm{CDCl}_{3}+\mathrm{DMSO}-\right.$ $\left.\mathrm{d}_{6}\right), 6.76(2 \mathrm{H}, \mathrm{dd}, \mathrm{CH}), 6.13(1 \mathrm{H}, \mathrm{s}, \mathrm{N}-\mathrm{H}), 5.35(1 \mathrm{H}$, $\mathrm{s}, \operatorname{Ar}-\mathrm{OH}), 7.10-8.02(\mathrm{~m}, 8 \mathrm{H}, \mathrm{Ar}-\mathrm{H})$; Analysis $\left(\mathrm{C}_{15} \mathrm{H}_{12} \mathrm{O}_{4} \mathrm{~N}_{2}\right)$ cal. (found) \%: C; 63.38 (63.42), H; 4.25 (4.52), N; 9.85(9.53). MS: (m/z) $284\left(\mathrm{M}^{+}\right) .1 \mathrm{~d}$ $\left(\mathrm{R}=\mathrm{p}-\mathrm{OCH}_{3}-\mathrm{C}_{6} \mathrm{H}_{4}\right):-\mathrm{IR}\left(\mathrm{KBr}, \mathrm{cm}^{-1}\right): 3431(\mathrm{Ar}-\mathrm{OH}$ str.), 3211 ( $\mathrm{NH}$ str.), 2831 ( $\mathrm{CH}_{2}$ str), 1493(C=C str), 1101(C-O-C str); ${ }^{1} \mathrm{HNMR}(\delta \mathrm{ppm})\left(\mathrm{CDCl}_{3}+\mathrm{DMSO}-\right.$ $\left.\mathrm{d}_{6}\right), 6.76(2 \mathrm{H}, \mathrm{dd}, \mathrm{CH}), 6.14(1 \mathrm{H}, \mathrm{s}, \mathrm{N}-\mathrm{H}), 5.35(1 \mathrm{H}$, $\mathrm{s}, \quad$ Ar- $\mathrm{OH}), \quad 7.10 \quad-8.01(\mathrm{~m}, \quad$ Ar-H $) ;$ Analysis $\left(\mathrm{C}_{16} \mathrm{H}_{15} \mathrm{ON}_{3}\right)$ cal. (found) \%: C; 71.36 (71.57), H; 5.61(6.02), N; 5.26 (4.99); MS (m/z): $269\left(\mathrm{M}^{+}\right) .1 \mathrm{e}$ $\left(\mathrm{R}=2-\mathrm{OH}-\mathrm{C}_{6} \mathrm{H}_{4}\right)$ :- $\mathrm{IR}\left(\mathrm{KBr}, \mathrm{cm}^{-1}\right): 3312(\mathrm{Ar}-\mathrm{OH}$ str.), 3208(NH str.), 2834( $\mathrm{CH}_{2}$ str.), $1505(\mathrm{C}=\mathrm{C}$ str.); ${ }^{1} \mathrm{HNMR}(\delta \mathrm{ppm})\left(\mathrm{CDCl}_{3}+\mathrm{DMSO}_{6}\right): 5.18(1 \mathrm{H}, \mathrm{s}$, Ar-OH), $5.35(1 \mathrm{H}, \mathrm{s}, \mathrm{Ar}-\mathrm{OH}), 6.56(2 \mathrm{H}, \mathrm{dd}, \mathrm{CH})$, $6.23(1 \mathrm{H}, \mathrm{s}, \mathrm{N}-\mathrm{H}), 7.10-8.00(\mathrm{~m}, 8 \mathrm{H}, \mathrm{Ar}-\mathrm{H})$; Analysis $\left(\mathrm{C}_{15} \mathrm{H}_{13} \mathrm{O}_{3} \mathrm{~N}\right)$ cal. (found) \%: C; 70.58 (70.82), H; 5.13(5.34), N; 5.49 (5.26); MS (m/z): $255\left(\mathrm{M}^{+}\right)$. $1 f\left(\mathrm{R}=\mathrm{p}-\mathrm{Cl}-\mathrm{C}_{6} \mathrm{H}_{4}\right)$ :- IR $\left(\mathrm{KBr}, \mathrm{cm}^{-1}\right): 3417$ (Ar-OH str.), 3278 (NH str.), 2932 (C-H str.), 2836 $\left(\mathrm{CH}_{2}\right.$ str.), $742(\mathrm{C}-\mathrm{Cl}),{ }^{1} \mathrm{HNMR}(\delta \mathrm{ppm})\left(\mathrm{CDCl}_{3}+\right.$ DMSO-d $\left._{6}\right), 6.76(2 \mathrm{H}, \mathrm{dd}, \mathrm{CH}), 6.21(1 \mathrm{H}, \mathrm{s}, \mathrm{N}-\mathrm{H})$, $5.35(1 \mathrm{H}, \mathrm{s}, \mathrm{Ar}-\mathrm{OH}), 7.19-8.00(\mathrm{~m}, 8 \mathrm{H}, \mathrm{Ar}-\mathrm{H})$. Analysis $\left(\mathrm{C}_{15} \mathrm{H}_{12} \mathrm{O}_{2} \mathrm{NCl}\right)$ cal. (found) \%: $\mathrm{C} ; 65.82$ (66.12), H; 4.42 (4.63), N; 5.12 (5.43); MS (m/z): 273( $\left.\mathrm{M}^{+}\right)$.

Preparation of 4-[(5'-phenyl-4',5'-dihydroisoxazol-3'yl-amino] phenol 2a. A mixture of compound 1a $(0.01 \mathrm{~mol})$ and hydroxylamine hydrochloride $(0.01 \mathrm{~mol})$ in ethanol $(30 \mathrm{ml})$ was refluxed on a water bath for $6 \mathrm{~h}$. The reaction mixture was concentrated under vacuum, cooled and poured into ice-cold water. The solid 2a thus separated was filtered, dried and recrystallized from ethanol. Compounds 2b-f was prepared similarly. Melting points, yields and molecular formulas are given in Table 1. 2a $\left(\mathrm{R}=-\mathrm{C}_{6} \mathrm{H}_{5}\right)$ :- IR $\left(\mathrm{KBr}, \mathrm{cm}^{-1}\right)$ : $3460(\mathrm{Ar}-$ OH str.), 3295 (NH str.), 3028 (C-H str.), 1630 (C=N str.), 1255 (C-O str.) ${ }^{1} \mathrm{HNMR}(\delta \mathrm{ppm})\left(\mathrm{CDCl}_{3}+\right.$ DMSO-d $\left.\mathrm{d}_{6}\right), 3.20(1 \mathrm{H}, \mathrm{t}, \mathrm{CH}), 6.11(1 \mathrm{H}, \mathrm{s}, \mathrm{N}-\mathrm{H}), 4.30$ $\left(2 \mathrm{H}, \mathrm{d}, \mathrm{CH}_{2}\right), 5.10(1 \mathrm{H}, \mathrm{s}, \mathrm{Ar}-\mathrm{OH}), 6.79-8.01(\mathrm{~m}$, $9 \mathrm{H}, \mathrm{Ar}-\mathrm{H})$; Analysis $\left(\mathrm{C}_{15} \mathrm{H}_{14} \mathrm{~N}_{2} \mathrm{O}_{2}\right)$ cal. (found) $\%$ : $\mathrm{C} ; 70.85(70.45), \quad \mathrm{H} ; 5.55(5.76), \mathrm{N} ; 11.02(11.29)$. MS:(m/z): 254(M $\left.\mathrm{M}^{+}\right)$. $2 \mathrm{~b}$ ( $\mathrm{R}=$ Furyl):- IR $\left(\mathrm{KBr}, \mathrm{cm}^{-1}\right)$ : 3309 (Ar-OH str.), 3260 (NH str.), 3065 (O-H str.), $2927\left(\mathrm{CH}_{2}\right.$ str. $), 1630$ (C=N str.), 1479 (C=C str.), 1229 (C-O str.), 1137 (C-O-C str.); ${ }^{1} \mathrm{HNMR}(\delta \mathrm{ppm})$ $\left(\mathrm{CDCl}_{3}+\mathrm{DMSO}_{-} \mathrm{d}_{6}\right), 3.13$ (3H, m, CH furyl), 4.41 (d, $2 \mathrm{H}, \mathrm{CH}_{2}$ ioxazoline), $5.32(1 \mathrm{H}, \mathrm{s}, \mathrm{Ar}-\mathrm{OH}), 6.24$ $(1 \mathrm{H}, \mathrm{d}, \mathrm{N}-\mathrm{H}), 6.62(1 \mathrm{H}, \mathrm{t}, \mathrm{CH}$ ioxazoline $), 6.82-7.88$ (m, $4 \mathrm{H}$, Ar- $\mathrm{H})$; Analysis $\left(\mathrm{C}_{13} \mathrm{H}_{12} \mathrm{~N}_{2} \mathrm{O}_{3}\right)$ cal. (found) \%: C; 63.93 (64.23), H; 4.95 (4.56), N; 11.47 (11.63). MS (m/z): $244\left(\mathrm{M}^{+}\right) ; 2 \mathrm{c}\left(\mathrm{R}=\mathrm{p}-\mathrm{NO}_{2}-\mathrm{C}_{6} \mathrm{H}_{4}\right)$ :- $\mathrm{IR}(\mathrm{KBr}$, $\mathrm{cm}^{-1}$ ): 3499 (Ar-OH str.), 3298 (NH str.), 3091 (C-H str.), 2841 ( $\mathrm{CH}_{2}$ str.), 1630 (C = N str.), 1560 (C$\mathrm{NO}_{2}$ asym. str.), 1489 (C=C str.), 1235 (C-O str.); ${ }^{1} \mathrm{HNMR}(\delta \mathrm{ppm})\left(\mathrm{CDCl}_{3}+\mathrm{DMSO}_{-} \mathrm{d}_{6}\right), 6.23(1 \mathrm{H}, \mathrm{s}$, $\mathrm{N}-\mathrm{H})$, 4.01- $4.14\left(2 \mathrm{H}, \mathrm{d}, \mathrm{CH}_{2}\right), 5.26(1 \mathrm{H}, \mathrm{s}, \mathrm{Ar}-\mathrm{OH})$, $6.73(1 \mathrm{H}, \mathrm{t}, \mathrm{CH}), 7.22-8.21$ ( m, 4H, Ar-H ); Analysis $\left(\mathrm{C}_{15} \mathrm{H}_{13} \mathrm{~N}_{3} \mathrm{O}_{4}\right)$ cal. (found) \% : C; $60.20(60.43), \mathrm{H}$; 4.38 (4.87), N; 14.04 (14.43). MS (m/z): $299\left(\mathrm{M}^{+}\right)$. 2d $\left(\mathrm{R}=\mathrm{p}-\mathrm{OCH}_{3}-\mathrm{C}_{6} \mathrm{H}_{4}\right):-\mathrm{IR}\left(\mathrm{KBr}, \mathrm{cm}^{-1}\right): 3436(\mathrm{Ar}-$ OH str.), 3207 (NH str.), 3045 (C-H str), $2831\left(\mathrm{CH}_{2}\right.$ str.), 1617 ( $\mathrm{C}=\mathrm{N}$ str), 1499 ( $\mathrm{C}=\mathrm{C}$ str), 1223 (C-O str), 1093 (C-O-C str); ${ }^{1} \mathrm{HNMR}(\delta \mathrm{ppm})\left(\mathrm{CDCl}_{3}+\right.$ DMSO-d $\left.\mathrm{d}_{6}\right): 3.83\left(\mathrm{~s}, 3 \mathrm{H}, \mathrm{OCH}_{3}\right), 4.38\left(2 \mathrm{H}, \mathrm{d}, \mathrm{CH}_{2}\right)$, $5.43(1 \mathrm{H}, \mathrm{s}, \mathrm{Ar}-\mathrm{OH}), 6.15(1 \mathrm{H}, \mathrm{s}, \mathrm{N}-\mathrm{H}), 6.73(1 \mathrm{H}, \mathrm{t}$, $\mathrm{CH}), \quad 7.10-8.05 \quad(\mathrm{~m}, \quad 8 \mathrm{H}, \quad$ Ar-H $)$; Analysis $\left(\mathrm{C}_{16} \mathrm{H}_{16} \mathrm{~N}_{2} \mathrm{O}_{3}\right)$ cal. (found) \% : C; 67.59 (68.01), $\mathrm{H}$; 5.67 (5.33), N; 9.85 (10.12). MS: (m/z) $284\left(\mathrm{M}^{+}\right) .2 \mathrm{e}$ ( $\left.\mathrm{R}=2-\mathrm{OH}-\mathrm{C}_{6} \mathrm{H}_{4}\right)$ :- IR $\left(\mathrm{KBr}, \mathrm{cm}^{-1}\right): 3309$ (Ar-OH str.), 3201 ( $\mathrm{NH}$ str.), 2841 ( $\mathrm{CH}_{2}$ str.), 1630 (C=N str.), 1505 (C=C str.), 1235 (C-O str.); ${ }^{1} \mathrm{HNMR}(\delta$ ppm) $\left(\mathrm{CDCl}_{3}+\mathrm{DMSO}_{-} \mathrm{d}_{6}\right): 4.21\left(2 \mathrm{H}, \mathrm{d}, \mathrm{CH}_{2}\right), 5.42(1 \mathrm{H}, \mathrm{s}$, Ar-OH), 5.48 (1H, s, Ar-OH), 6.21(1H, s, N-H), 6.59 $(1 \mathrm{H}, \mathrm{t}, \mathrm{CH}), 7.12-8.11(\mathrm{~m}, 8 \mathrm{H}, \mathrm{Ar}-\mathrm{H})$. Analysis $\left(\mathrm{C}_{15} \mathrm{H}_{14} \mathrm{~N}_{2} \mathrm{O}_{3}\right)$ cal.(found) \% : C; 66.66 (66.24), H; 5.22 (5.47), N; 10.36 (10.01). MS (m/z): $270\left(\mathrm{M}^{+}\right) ; 2 f$ $\left(\mathrm{R}=\mathrm{p}-\mathrm{Cl}-\mathrm{C}_{6} \mathrm{H}_{4}\right)$ :- IR $\left(\mathrm{KBr}, \mathrm{cm}^{-1}\right): 3412$ (Ar-OH str.), 3276 (NH str.), 2921 (C-H str.), 2826 ( $\mathrm{CH}_{2}$ str.), 1024 (C=N str.), 742 (C-Cl str.); ${ }^{1} \mathrm{HNMR}(\delta \mathrm{ppm})$ $\left(\mathrm{CDCl}_{3}+\mathrm{DMSO}_{-} \mathrm{d}_{6}\right): 4.31\left(2 \mathrm{H}, \mathrm{d}, \mathrm{CH}_{2}\right), 5.37(1 \mathrm{H}, \mathrm{s}$, Ar-OH), $6.25(1 \mathrm{H}, \mathrm{s}, \mathrm{N}-\mathrm{H}), 6.81(1 \mathrm{H}, \mathrm{t}, \mathrm{CH}), 7.24-$ $8.14(\mathrm{~m}, \quad 8 \mathrm{H}, \quad$ Ar- $\mathrm{H})$; Analysis $\left(\mathrm{C}_{15} \mathrm{H}_{13} \mathrm{~N}_{2} \mathrm{O}_{2} \mathrm{Cl}\right)$ 
cal.(found) \% : C; 62.40 (62.78), H; 4.54 (4.02), N; 9.70 (10.09); MS:(m/z): $288\left(\mathrm{M}^{+}\right)$.

\section{Biological evaluation}

Analgesic activity: The analgesic activity was determined by tail immersion method. ${ }^{12}$ Wistar albino mice $(\mathrm{n}=6)$ of either sex selected by random sampling technique were used for the study. Paracetamol at $(100 \mathrm{mg} / \mathrm{kg})$ was administered as standard drug for comparison. The test compounds $(100 \mathrm{mg} / \mathrm{kg})$ were administered orally by intragastric tube. The animals were held in position by a suitable restrained with the tail extending out and the tail (up to $5 \mathrm{~cm}$ ) was then dipped in a beaker of water maintained at $55 \pm 5{ }^{\circ} \mathrm{C}$. The time in seconds taken to withdraw the tail clearly out of water was taken as the reaction time. The reading was recorded at 30,60, 120 and $180 \mathrm{~min}$ after administration of compounds. A cut off point of $10 \mathrm{sec}$ was observed to prevent the tail damage. The results are presented in Table 2.

Antimicrobial activity: In vitro antimicrobial study was carried on Muller Hinton agar (Hi-media) plates $\left(37^{\circ} \mathrm{C}, 24 \mathrm{~h}\right)$ by agar diffusion cup plate method (13). The test microorganisms were obtained from Department of Microbiology, OUAT, Orissa, India. All the compounds were screened for antimicrobial activity at $100 \mu \mathrm{g} / \mathrm{ml}$ concentration level against the following bacterial Strains: Staphylococcus aureus, Escherichia coli, and
Salmonella typhi. Antifungal activity was tested on Sabouraud dextrose agar (Himedia) plates $\left(26^{\circ} \mathrm{C}, 48\right.$ $72 \mathrm{~h}$ ) by cup plate method against Candida albicans and Aspergillus niger at a concentration level of 100 $\mu \mathrm{g} / \mathrm{ml}$. Ciprofloxacin and clotrimazole were used as reference standard for comparison of antibacterial and antifungal activity. DMSO was used as a solvent control for both antibacterial and antifungal activities. The results are presented in Table 3.

\section{RESULTS AND DISCUSSION}

Biological results are reported in Tables 2 and 3, which also records the effects of standard drugs included for comparison. Series of compounds were prepared in this study, exhibited significant pharmacological properties in different biological models. The general pattern of pharmacological activity encountered with these synthesized compounds were seen mainly for their effect on pain perception. However, there was a moderate, well defined antimicrobial activity associated with many of these compounds. Considerable variation of these effects were seen with each structural changes, varying from agents that had less activity to those with high potency, and significant changes in potency resulted even from minor change in chemical structure as shown in Table 2 and 3.

Table 2. Screening of analgesic activity of compounds 2a-f by tail immersion method

\begin{tabular}{|c|c|c|c|c|c|}
\hline \multirow{2}{*}{$\frac{\text { Compound }}{\text { Code }}$} & \multirow{2}{*}{$\frac{\text { Dose }}{(\mathrm{mg} / \mathrm{kg})}$} & \multicolumn{4}{|c|}{ Percentage of analgesic activity } \\
\hline & & $30 \mathrm{~min}$. & 1 hour & 2 hour & 3 hour \\
\hline $2 \mathbf{a}$ & 100 & $30 \pm 0.38^{*}$ & $32 \pm 0.72^{*}$ & $37 \pm 0.47^{*}$ & $28 \pm 0.91^{*}$ \\
\hline $2 \mathbf{b}$ & 100 & $36 \pm 0.28^{*}$ & $41 \pm 0.45^{* *}$ & $44 \pm 0.49^{*}$ & $36 \pm 0.26^{*}$ \\
\hline 2c & 100 & $40 \pm 0.52^{* *}$ & $44 \pm 0.23^{* *}$ & $49 \pm 0.21^{* *}$ & $36 \pm 0.29^{*}$ \\
\hline 2d & 100 & $45 \pm 0.22^{*}$ & $50 \pm 0.22^{* *}$ & $57 \pm 0.43^{*}$ & $47 \pm 0.27^{*}$ \\
\hline $2 e$ & 100 & $39 \pm 0.26^{*}$ & $43 \pm 0.31^{* *}$ & $47 \pm 0.32^{* *}$ & $36 \pm 0.44^{* *}$ \\
\hline $2 f$ & 100 & $41 \pm 0.46^{* *}$ & $45 \pm 0.61^{* *}$ & $51 \pm 0.52^{* *}$ & $37 \pm 0.22^{* *}$ \\
\hline Paracetamol & 100 & $38 \pm 0.42^{* *}$ & $47 \pm 0.82^{* *}$ & $52 \pm 0.71^{* *}$ & $33 \pm 0.31^{* *}$ \\
\hline
\end{tabular}

Results are expressed in mean $\pm \operatorname{SEM}(\mathrm{n}=6)$ significance levels $* \mathrm{P}<0.05,{ }^{* *} \mathrm{P}<0.01$ and ${ }^{* * *} \mathrm{P}<0.001$ as compared with the respective control. 
Table 3. Antibacterial and antifungal activity of compounds 2a-f

\begin{tabular}{cccccccc}
\hline Compound & Conc. $(\mu \mathrm{g} / \mathrm{ml})$ & \multicolumn{5}{c}{ Zone of inhibition $(\mathrm{mm})$} \\
\cline { 3 - 8 } & 100 & S. $a$ & S. $f$ & E. $c$ & S. $t$ & C. $a$ & A. $n$ \\
\hline 2a & 100 & 12 & 16 & 11 & 14 & 08 & 12 \\
2b & 100 & 14 & 13 & 15 & 12 & 14 & 13 \\
2c & 100 & 22 & 20 & 21 & 19 & 17 & 19 \\
2d & 100 & 13 & 13 & 12 & 14 & 12 & 11 \\
2e & 100 & 19 & 17 & 16 & 19 & 14 & 17 \\
2f & 100 & 21 & 22 & 21 & 18 & 21 & 24 \\
Ciprofloxacin & 10 & 29 & 31 & 32 & 26 & - & - \\
Clotrimazole & 20 & - & - & - & - & 28 & 27 \\
\hline
\end{tabular}

*Average of three readings

S. a: Staphylococcus aureus; S. f: Staphylococcus fecalis; E. c: Escherichia coli; S. t: Salmonella typhi; C. a: Candida albicans; A. n: Aspergillus niger

Analgesic activity. Few of the compounds among the tested compounds 2a-2f exhibited significant activity in experimental model used. Particularly interests are the results obtained in the Glassman's procedure, which utilizes selective inhibition of inflammatory pain as a model for screening anti-inflammatory drugs. On comparing the structure of synthesized compounds, it appeared that a p-nitrophenyl, p-methoxyphenyl or pcholorophenyl 2c, $2 \mathbf{e}$ and $2 \mathbf{f}$ substituents at 5 position of isoxazole ring resulted in remarkable increase in analgesic activity.

Antimicrobial activity. The in vitro antimicrobial activity of compounds 2a-2f were determined by agar cup plate method, the results of which are summarized in Table 3.The antimicrobial data in Table 3 clearly indicated that the halogen, nitro and hydroxyphenyl substituents at 5 position of isoxazole ring were by far the most active substitutents. The methoxy group generally confered week antimicrobial activity. Phenyl and furyl substitutions are weakly active to inactive among the synthesized compounds. The compounds 2c, $2 \mathbf{e}$ and 2f showed significant activity against $S$. aureus and S. typhi; the compounds $\mathbf{2 c}$ and $\mathbf{2 f}$ exhibited promising activity against $C$. albicans and $A$. niger. However, the entire tested compounds were found to be less active as antibacterial and antifungal agents in comparison to ciprofloxacin and clotrimazole, respectively.
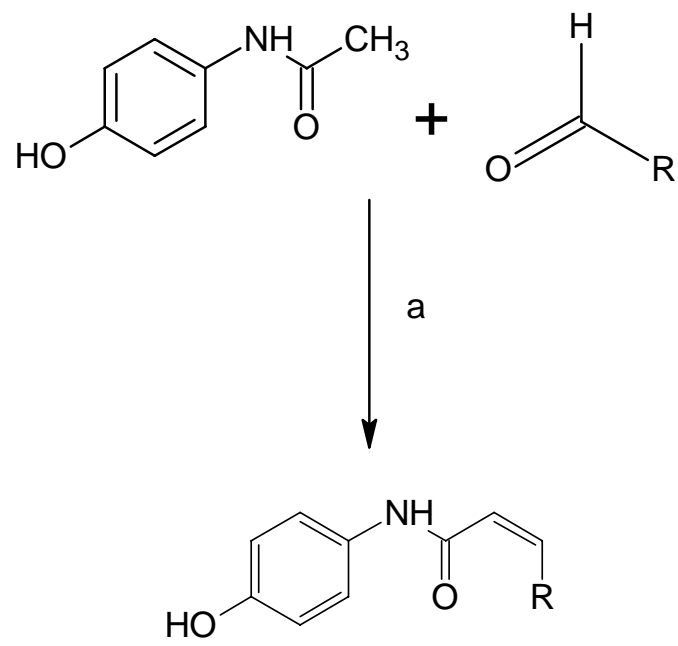

1a-f

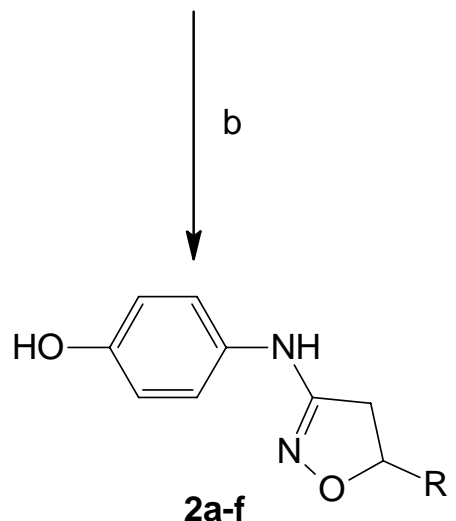

$\mathrm{R}=-\mathrm{C}_{6} \mathrm{H}_{5},-2-$ Furyl, $-4-\mathrm{NO}_{2}-\mathrm{C}_{6} \mathrm{H}_{4},-4-\mathrm{OCH}_{3}-\mathrm{C}_{6} \mathrm{H}_{4},-2-\mathrm{OH}-\mathrm{C}_{6} \mathrm{H}_{4},-$ $4-\mathrm{Cl}-\mathrm{C}_{6} \mathrm{H}_{4}$

Scheme 1. a) Ethanol, $2 \% \mathrm{NaOH}, 50-60{ }^{\circ} \mathrm{C}$; b) Ethanol, Hydroxylamine hydrochloride 


\section{CONCLUSION}

The purpose of the present study was to examine whether molecular modification might result in detection of new potential antimicrobial and analgesic drugs. A series of compounds were prepared and assayed in a variety of biological test for analgesic and antimicrobial activity. The data reported in Tables 2 and 3 showed that effect of variation in chemical structure on activity was rather unpredictable. Seldom a particular structural modification led to uniform alteration in activity in all tests. However, some points of interest did emerge and a few generalizations can be made. The substitution which appeared to be most important for high order of activity in the greatest number of test was the p-choloroaryl group. The substitution of $p$ nitrophenyl and p-hydroxyphenyl group at 5 position of isoxazole ring resulted in compounds $\mathbf{2 c}$ and $\mathbf{2 e}$, respectively with potent analgesic and antimicrobial activities. Obviously, the comparative evaluation of the active compounds will require further studies. The data reported in this article may be a helpful guide for the medicinal chemists working in the area.

\section{ACKNOWLEDGEMENT}

The authors would like to express their gratitude and thanks to the Head, University Dept. of Pharmaceutical Sciences, Utkal University and Institute of Pharmacy and Technology, Salipur, Cuttack for necessary facilities to carry out this research work.

\section{REFERENCES}

1. Rani, P., Srivastava, V.K. and Kumar, A. 2003. Isoxazolinyl Derivatives of Anthranilic Acid as Anti-Inflammatory Agents. Ind. J. Chem. 42B, 1729-1733.

2. Habeeb, G.A., Rao, P.N.P. and Kanus, E.E. 2001. Design and Synthesis of 4,5-Diphnyl-4-isoxazoline: Novel Inhibitors of Cyclooxygenase-2 with Analgesic and Anti-inflammatory Activity. J. Med. Chem. 44, 2921-2927.
3. Habeeb, G.A., Rao, P.N.P. and Kanus, E.E. 2000. Design and Synthesis of 4,5-Diarylisoxazoles: Novel Inhibitors of Cyclooxygenase-2(cox-2) with Analgesic and Antiinflammatory Activity. Drug Dev. Res. 51, 273-286.

4. Safak, C., Erdojjan, I.H,, Palaska, T.E., Sunal, T.R. and Durd. 1992. Synthesis of 3-(Pyridylethyl) Benzoxazolinone Derivatives:Potent Analgesic and Anti-inflammatory Compounds Inhibiting Prostaglandin E2. J. Med. Chem, 5, 1296-1299.

5. Goda, F.E., Maarouf, A.R, and EL-Bendary, E.R. 2003. "Synthesis and Antimicrobial Evaluation of New Isoxazole and Pyrazole Derivaties." S. Pharm. Journal. 11, 111-117.

6. Diadone, G., Raffa, D., Maggio, B., Plescia, F., Curuli, VMC., Mangano, N.G. and Caruso, A. 2007. Synthesis and Pharmacological Activities of Novel-3-(Isoxazol-3-yl)Quinazolin-4(3H)-one. Archive Pharmazie. 340A, 163-164.

7. Imran, M. and Khan, S.A. 2004. Synthesis of 3,5Disubstituted Isoxazoles as Antibacterial and Antifungal agents. Indian J. Heterocycl. Chem. 13, 213-216.

8. Naik, S.M. and Naik, HB. 1998. Synthesis and antibacterial activity of some chalcones and isoxazoles. Oriental J. Chem. 14, 167-1671.

9. Yasuda, N., Iwagami, H. and Sasaki, Y. 1983. Synthesis and Antibacterial Activity of Triazole and Isoxazoles Derivatives of Ampicillin. J. Antibiot (Tokyo). 36(11), 1516-24.

10. Merja, B.C., Joshi, A.M., Parikh, K.A. and Parikh, A.R. 2004. Synthesis and Biological Evaluation of Pyrido (1,2-a) Pyrimidine and Isoxazoline Derivatives. Ind. J. Chem. 43B, 909-912.

11. Sahu, S.K., Mishra, S.K, Banerjee, M, Panda. P.K. and Mishro, P.K. 2006. Synthesis, Partition Coefficient and Antibacterial Activity of 3' aryl-6-phenyl (substituted) -cis5 'a,6'-dihydrospiro [3H-indole-3,4'thiozolo $\quad\left(5^{\prime}, 1^{\prime}\right.$ 'c)isoxazolo-2(1H)-ones]. J. Indian Chem. Soc. 83, 725-727.

12. Glassman, J.M. 1971. Agents with Analgesic Activity and Dependences Liability. In: Turner R. A. and Hebborn P. (Ed) Screening Methods in Pharmacology, Academic Press, New York and London. pp. 231-232.

13. Berry, A. (Eds) 1991. In: Antibiotics In Laboratory Medicine, $5^{\text {th }}$ edn, William and Willkisns, Baltimore, MD., 1. 